\title{
Tragus formation during concha-type microtia repair using a chondrocutaneous island flap
}

\author{
Jeong Hyun $\mathrm{Ha}^{1}$, \\ Euicheol Jeong ${ }^{2}$, \\ Hudson Lazaro ${ }^{3}$ \\ ${ }^{1}$ Department of Plastic and Reconstructive \\ Surgery, Seoul National University Hospital, \\ Seoul National University College of Medicine, \\ Seoul; \\ ${ }^{2}$ Department of Plastic Surgery, SMG-SNU \\ Boramae Medical Center, Seoul, Korea; \\ ${ }^{3}$ Cirurgia Plástica Transplante Capilar, Pará \\ de Minas, Brasil
}

\begin{abstract}
Formation of an ideally-shaped tragus remains one of the most challenging issues during staged tragus reconstruction in microtia patients. The authors describe a new method used to treat a unique case of concha-type microtia in which the 10 -year-old male patient had only a portion of pre-existing cartilage at the tragus site. An anomalous skin lump was also present. During the initial stages of the reconstruction, the two-stage Nagata method was used for surgical correction of the microtia. An autologous rib cartilage graft was used to form the ear framework. A temporoparietal fascia flap was also constructed. Remnant skin tags and anomalous cartilage that accompany microtia are usually removed during microtia repair. However, the cartilage and skin lump were preserved during the reconstruction. The skin lump was later used to form a vascularized chondrocutaneous island flap that supplemented the portion of cartilage during tragus formation. The result was a new tragus that was satisfactorily improved in both size and shape. Patients with concha-type microtia may benefit from the use of this new method for tragus formation.
\end{abstract}

Keywords: Congenital microtia / Surgical flaps / Ear auricle

\section{INTRODUCTION}

Microtia frequently accompany anomalous remnant skin tags, with or without the presence of a cartilage structure. The incidence of this condition is unknown. Abnormal skin tags and anomalous cartilage are usually removed during the staged reconstruction used for microtia repair. In most cases, the tragus is reconstructed separately [1-4].

Various surgical techniques are used for tragus reconstruction in microtia patients. Since Tanzer [1] first reported an ear reconstruction method for microtia repair using an autogenous rib cartilage graft, many plastic surgeons have also developed other techniques. However, formation of an ideally-shaped tragus remains a difficult issue. Use of a cartilage graft from the contralateral ear, an anteriorly-based conchal flap doubled upon itself (Kirkham

\section{Correspondence: Euicheol Jeong}

Department of Plastic Surgery, SMG-SNU Boramae Medical Center, 20 Boramae-ro 5-gil, Dongjak-gu, Seoul 07061, Korea

E-mail: ejeong.md@gmail.com

Received March 28, 2017 / Revised February 9, 2018 / Accepted February 24, 2018 method) by an extra cartilage piece attached to this main framework, or included in the main ear framework through an arched intertragal notch (Nagata method), are methods that have been reported [2-4].

Regardless of the technique used, postoperative flattening during long-term follow-up is caused by decreased blood flow in the transferred graft and flap, which are attached in a scarred area using staged surgery. Simultaneous cartilage implantation as a part of a one-stage framework formation results in a relatively good outcome because it is performed in an unscarred area. However, this method requires a relatively large amount of rib cartilage and technically demanding, time-consuming, formation of the framework. Recently, using remnant ear for tragus reconstruction is also introduced. Xiao et al. [5] reported W-shaped skin flap, conchal trace modification, or composite graft using remnant tissue not discarded from the previous stage. Here, we present a modification of staged tragus formation in which we used the anomalous skin lump and cartilage to make the new tragus. 


\section{IDEA}

A 10-year-old boy visited our clinic with a chief complaint of a small, right-sided ear deformity. The patient presented with a concha-type microtia (Fig. 1). Using the two-stage Nagata method, the patient underwent surgical correction of the microtia via an autologous rib cartilage graft to form the ear framework, and a temporoparietal fascia flap [4]. During the first stage, lower part was preserved since it resembled normal structure. The cartilage framework only included upper part of ear and concha area without tragus.

Additional minor surgery (third stage) was performed under local anesthesia to improve the shape of the tragus. The anomalous skin lump above the lobule and the underdeveloped traguslike structure containing the small cartilage were still present after the microtia surgery (Fig. 2). The outline of a $1.0 \times 0.9-\mathrm{cm}$ chondrocutaneous island flap was defined on the preserved anomalous skin lump. A small, 1-cm, scalpel incision was made above the underdeveloped tragus, and further dissection around the anomalous cartilage was performed. A tunnel was made between the tragus and the skin lump. The harvested island flap, which preserved the anteriorly based subcutaneous pedicle, was passed

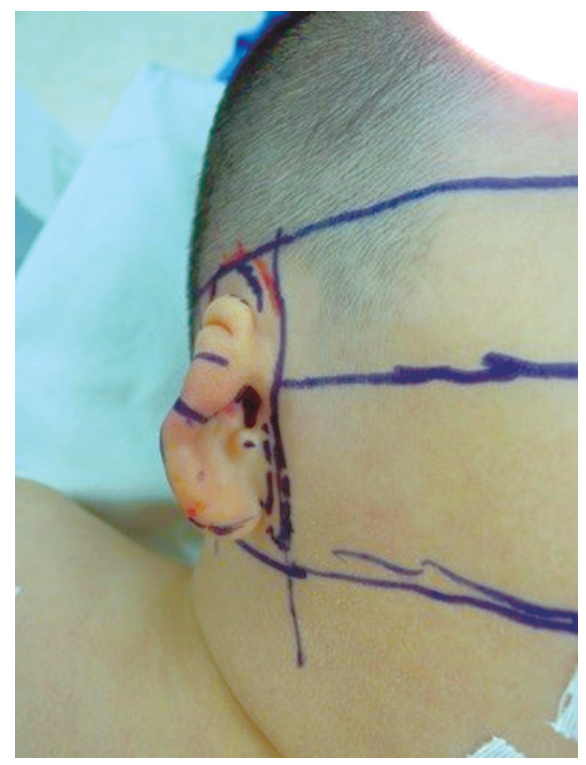

Fig. 1. A right-sided concha-type microtia with a small underdeveloped tragus, before surgery.

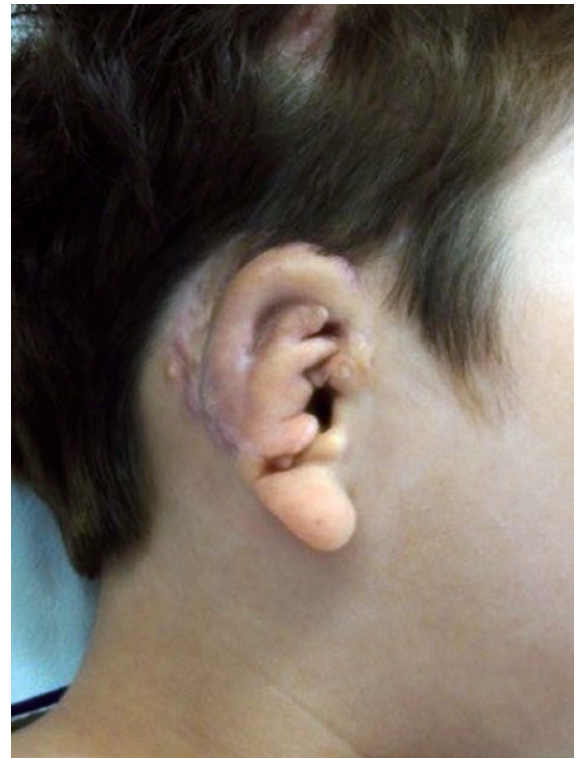

Fig. 2. After the procedure using the Nagata method for concha-type microtia repair, the anomalous skin lump above the lobule and facing the underdeveloped tragus can be observed.

through the tunnel and inserted up to the dissected area (Figs. 3, 4). The flap was closed using vicryl (6-0) suture material to add bulky soft tissue to the underdeveloped tragus. Vicryl (6-0) suture material was also used to close the donor site. An interrupted suture pattern was used for both closures. The wound healed uneventfully and the new tragus was satisfactorily improved in both size and shape. At 7-month follow-up, the size and shape of tragus were well maintained, and the patient was satisfied with the result (Fig. 5).

\section{DISCUSSION}

Tragus reconstruction is significant to ameliorate the aesthetic outcome in auricular reconstruction. Moreover, it is also related to improved functional outcome [6]. The tragus is present in some published case reports of concha-type microtia. Because the formed cartilage framework is usually used for the upper part of the deformed ear, simultaneous tragus reconstruction as a part of the framework is often unnecessary.

This unique case had only a portion of pre-existing cartilage at the tragus site, and an anomalous skin lump. The anomalous skin lump from the area adjacent to the tragus can be used as a chon- 

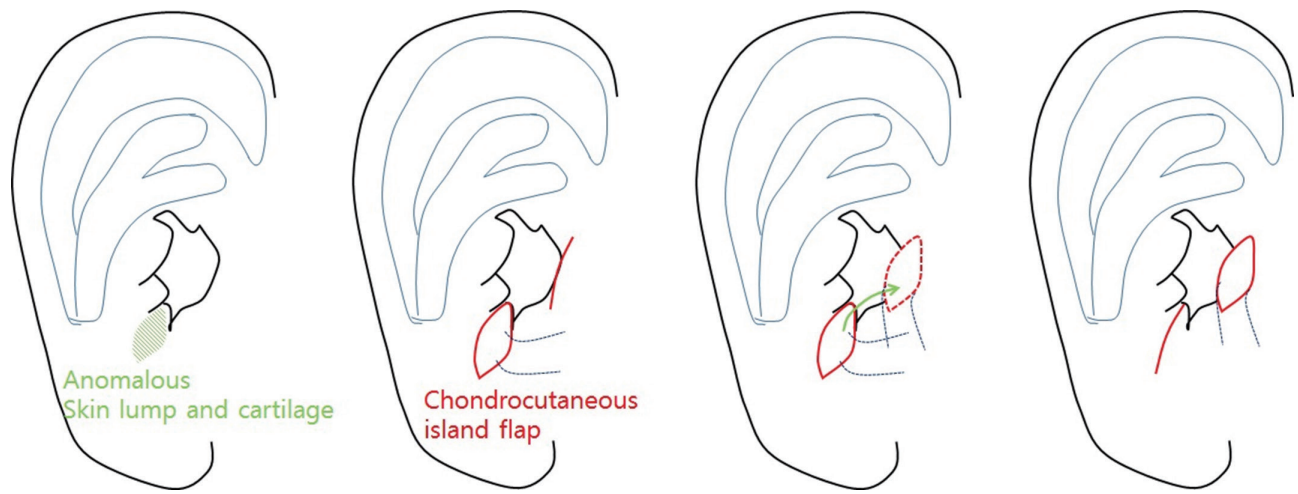

Fig. 3. Chondrocutaneous island flap method for tragus reconstruction.

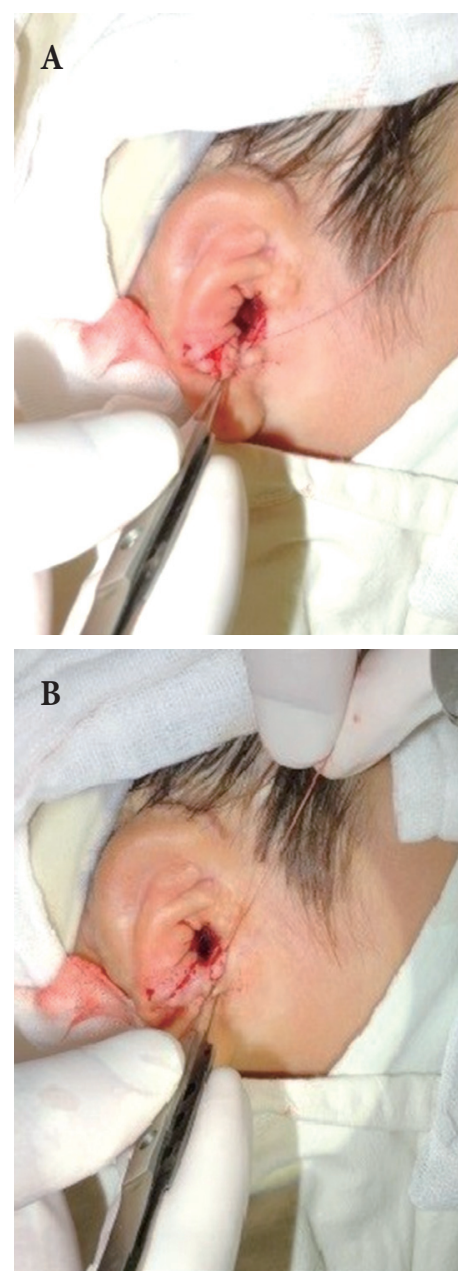

Fig. 4. (A) A subcutaneous pedicled chondrocutaneous island flap was prepared along the anomalous skin lump margin and a small $1-\mathrm{cm}$ vertical incision was made above the underdeveloped tragus. Further dissection around the anomalous cartilage was used to make the space filled by the flap. (B) Then the harvested island flap was passed through the tunnel and was used to fill the dissected area.

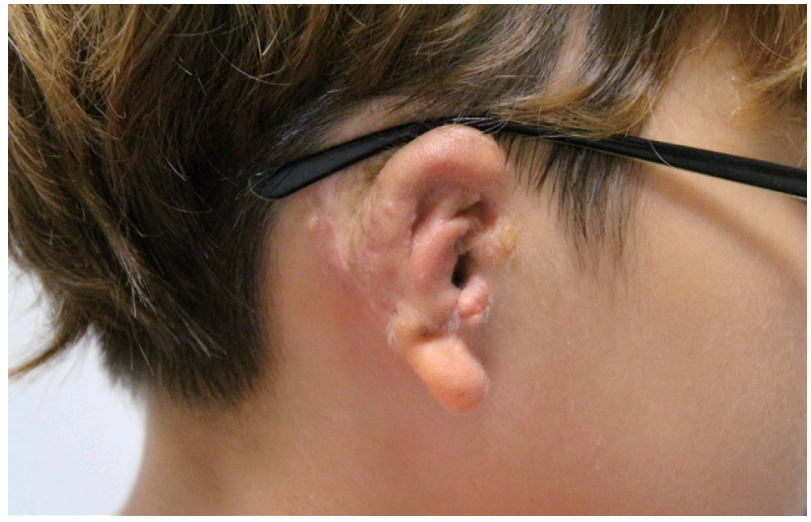

Fig. 5. The new tragus was well maintained in both size and shape at 7 months after surgery.

drocutaneous island flap based on dermal plexus, if it is not excised during previous stages of reconstruction. This method is possible because the ear is a highly vascularized tissue that allows the formation of a viable, random island flap [7]. Tragus reconstruction using remnant tissue has recently been tried $[8,9]$. Our case proves that, although it might not be possible in all cases, tragus reconstruction using anomalous tissue might be advantageous.

This method can be used for tragus formation in patients with a concha-type microtia. A chondrocutaneous island flap can be used for tragus formation in cases with an anomalous skin lump present at a location that is close enough in proximity to use an interpolation flap. 


\section{CONFLICT OF INTEREST}

No potential conflict of interest relevant to this article was reported.

\section{PATIENT CONSENT}

The patients provided written informed consent for the publication and the use of their images.

\section{REFERENCES}

1. Tanzer RC. Total reconstruction of the external ear. Plast Reconstr Surg Transplant Bull 1959;23:1-15.

2. Brent B. The correction of microtia with autogenous cartilage grafts: II. atypical and complex deformities. Plast Reconstr Surg 1980;66:1321.
3. Kirkham HL. The use of preserved cartilage in ear reconstruction. Ann Surg 1940;111:896-902.

4. Nagata S. Modification of the stages in total reconstruction of the auricle: part II. grafting the three-dimensional costal cartilage framework for concha-type microtia. Plast Reconstr Surg 1994;93:231-42.

5. Xiao Q, Shujie W, Hongxing Z, Haiyue J, Qinghua Y, Dashan Y. Using a remnant ear to reconstruct the tragus in total ear reconstruction. J Plast Reconstr Aesthet Surg 2009;62:1411-7.

6. Perez CF, Gaball CW. Functional and aesthetic tragal reconstruction in the age of mobile electronic devices. Case Rep Otolaryngol 2016;2016:2591705

7. Park C, Roh T. Button-down procedure for correction of cleft earlobe malformation. Plast Reconstr Surg 1997;99:1429-32.

8. Park C. Reconstruction of congenital tragal malformations accompanied by dystopic cartilage growth (accessory tragus). Plast Reconstr Surg 2015;135:1681-91.

9. Kim JT, Kim YH, Kim SW. One-stage reconstruction of a conchatype microtia using remnant tissue. J Craniofac Surg 2012;23:1785-6. 